\title{
Vascular Hemorrhagic Disorder
}

National Cancer Institute

\section{Source}

National Cancer Institute. Vascular Hemorrhagic Disorder. NCI Thesaurus. Code C35595.

A hemorrhagic disorder that is caused by pathologic changes in the vasculature. 